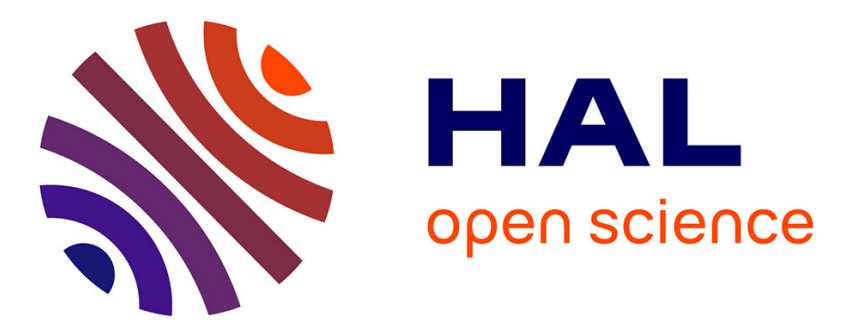

\title{
Cultures de l'information à l'université : savoirs en jeu, enjeux de savoir
}

\author{
Alexandre Serres
}

\section{To cite this version:}

Alexandre Serres. Cultures de l'information à l'université: savoirs en jeu, enjeux de savoir. Liquète, Vincent (sous la dir. de). Cultures de l'information, CNRS Editions, pp. 115-137, 2014, Les Essentiels d'Hermès, 978-2-271-08073-8. hal-02146046

\section{HAL Id: hal-02146046 https://hal.science/hal-02146046}

Submitted on 3 Jun 2019

HAL is a multi-disciplinary open access archive for the deposit and dissemination of scientific research documents, whether they are published or not. The documents may come from teaching and research institutions in France or abroad, or from public or private research centers.
L'archive ouverte pluridisciplinaire HAL, est destinée au dépôt et à la diffusion de documents scientifiques de niveau recherche, publiés ou non, émanant des établissements d'enseignement et de recherche français ou étrangers, des laboratoires publics ou privés.

\section{(ㅇ)(1) $\$$}

Distributed under a Creative Commons Attribution - NonCommercial - NoDerivatives| 4.0 
Serres, Alexandre. «Cultures de l'information à l'université ». In Liquète, Vincent (sous la dir. de). Cultures de l'information. Paris : CNRS Editions, 2014 (Collection Les Essentiels), p. $115-137$

\section{Cultures de l'information à l'université :}

\section{savoirs en jeu, enjeux de savoir}

Alexandre Serres

Inédit

Culture numérique, culture informatique, culture de l'information*, des réseaux, des médias, des TICe, des données, etc. : les appellations se bousculent, se croisent et se chevauchent pour désigner les nouvelles compétences, les nouveaux savoirs dont auraient besoin les étudiants, les doctorants, les enseignants-chercheurs, à l'heure des réseaux numériques. Dans cette prolifération terminologique, la «culture numérique » est devenue le terme générique, le mot-valise, englobant toutes les autres cultures et pratiques liées aux réseaux d'Internet. Mais un nouveau support de l'information suffit-il à caractériser toutes les réalités qu'il touche? Pourquoi ne pas parler plutôt de «cultures de l'information », pour souligner à la fois la multiplicité des cultures concernées par le phénomène informationnel et la prééminence de l'information sur le numérique, celui-ci pouvant être considéré comme l'une des catégories de l'information?

\section{Du singulier au pluriel}

La notion de culture de l'information présente une complexité intrinsèque, liée d'une part à la définition même du terme de «culture », d'autre part au deuxième terme de l'expression, «l'information», ainsi qu'au choix du singulier ou du pluriel. De quelle(s) catégorie(s) d'information les deux approches les plus courantes de la culture, sociologique et transmissive, sont-elles porteuses? L'information au sens des médias (l'info-news), au sens info-documentaire ou bibliothéconomique (l'info-knowledge) ou au sens informatique (l'infodata)? Des différences profondes, épistémologiques, séparent ces trois catégories, souvent confondues dans les esprits comme dans les référentiels de compétences. Une évolution 
notable marque la courte histoire des littératies* informationnelles en France, que l'on peut résumer en trois phases et trois problématiques :

- celle de la «maîtrise de l'information » (l'information literacy* des Anglo-saxons), basée sur une approche info-documentaire ou bibliothéconomique et sur des compétences procédurales (savoir chercher l'information, utiliser une bibliothèque, etc.) ; née à la fin des années 1970 aux États-Unis, la «maîtrise de l'information » a largement dominé les décennies 1980 et 1990, jusqu'aux années 2000 ;

- celle de la «culture de l'information », conçue de manière plus large et englobant à la fois des comportements personnels, des gestes cognitifs*, des valeurs, des pratiques, des savoirs théoriques, des savoir-faire, sur l'information au sens large ; cette problématique, qui émerge à la fin des années 1990 (Baltz, 1998), a notamment marqué de nombreux travaux des années 2000 ;

- celle de la «translittératie*» ou «des» cultures de l'information, qui s'emploie à appréhender simultanément les pratiques et cultures mobilisées dans nos interactions avec toutes les médiations d'information et de communication ${ }^{1}$; née aux États-Unis et en GrandeBretagne vers le milieu des années 2000, cette problématique fait aujourd'hui l'objet de nombreux débats et travaux de recherche en France. Cette dernière problématique, délibérément plurielle, entérine tout d'abord le dépassement des approches bibliothéconomiques de la formation des étudiants, qui gardent leur légitimité mais ne peuvent prétendre représenter tout le champ des cultures de l'information. Au-delà, ce sont même les approches purement informationnelles (au sens info-documentaire) qui sont bousculées et remises en cause par la notion de translittératie, qui cherche à clarifier les relations ${ }^{2}$ entre cultures informatique, médiatique et informationnelle et à prendre en compte la multiplicité des usages et des nouvelles compétences liés au numérique. Face à l'hybridation des cultures et des pratiques sur Internet, la translittératie se veut une réponse globale, transversale.

\footnotetext{
${ }^{1}$ Rappelons la définition de la translittératie par Sue Thomas (Thomas et al., 2007) : «l'habileté à lire, écrire et interagir par le biais d'une variété de plateformes, d'outils et de moyens de communication, de l'iconographie à l'oralité en passant par l'écriture manuscrite, l'édition, la télé, la radio et le cinéma, jusqu'aux réseaux sociaux ». Traduction par François Guité (sur son blog : 〈http://www.francoisguite.com/2007/12/la-translitteratie/>).

${ }^{2}$ Voir notamment, dans Les e-dossiers de l'audiovisuel de l'INA, le dossier consacré à «L'éducation aux cultures de l'information », janvier 2012. En ligne sur : http://www.ina-expert.com/e-dossiers-de-1-audiovisuel/edossier-de-l-audiovisuel-1-education-aux-cultures-de-l-information.html
} 


\section{Six cultures de l'information}

À l'université, au moins six types de cultures de l'information peuvent être recensées et distinguées, chacune porteuse de compétences, de savoirs et d'enjeux spécifiques. La plupart d'entre elles se caractérisent par une diffusion, un élargissement hors de leur cadre d'origine, que celui-ci soit professionnel ou scientifique. Et ce processus de circulation des cultures de l'information d'un univers à un autre, de percolation de leurs concepts, représente l'un de leurs enjeux de savoirs les plus importants. Ainsi la culture informatique n'est plus, de facto, l'apanage des seuls informaticiens, ni la culture info-documentaire celle des bibliothécaires ; mais comment renforcer et transformer, chez les étudiants et les doctorants, ces cultures de l'information acquises spontanément? Un autre enjeu de savoir est la multiplication de nouveaux référentiels de compétences, qui cherchent chacun à actualiser la liste des nouvelles compétences portées par ces cultures. Essayons de les passer en revue une à une.

\section{Des cultures documentaire et informationnelle, indispensables à la réussite des études}

Un premier ensemble regroupe les cultures documentaire et informationnelle, qui ont servi de matrice à la «maîtrise de l'information» historique (l'information literacy), et qui englobent à la fois l'usage averti des bibliothèques, des ressources électroniques et celui des outils et méthodes de recherche d'information.

Savoir utiliser les structures documentaires à l'université, leurs ressources, les bases de données documentaires, etc. : ces compétences relèvent d'une culture info-documentaire, dont la maîtrise représente un enjeu méthodologique essentiel pour la réussite des études, comme l'ont montré les travaux pionniers d'Alain Coulon (1999) sur «l'affiliation intellectuelle » des étudiants. Si cette culture documentaire, historiquement portée par les métiers des bibliothèques, n'a cessé d'évoluer en épousant les évolutions technologiques, elle n'en garde pas moins un certain nombre d'invariants, de notions clés pérennes.

La culture informationnelle*, plus large que le seul domaine des bibliothèques, concerne toutes les interactions avec les dispositifs d'accès à l'information, hors et sur le Web. Maîtrise de la recherche d'information, utilisation avertie des outils de recherche, usage et pratique de la veille, capacité à évaluer l'information trouvée sur le Web, connaissance et pratique des outils pour le traitement, la mémorisation, la diffusion de l'information, etc. : le champ des compétences, des pratiques et des savoirs couvert par cette culture informationnelle s'est considérablement élargi. Cette culture informationnelle ne saurait être limitée au seul usage des outils : elle se fonde d'abord sur un rapport maîtrisé à l'information 
en tant qu'élément de connaissance (l'info-knowledge), i.e. la capacité à rechercher, identifier, filtrer, évaluer, traiter l'information. Les compétences informationnelles* ont fait l'objet de nombreux référentiels depuis quinze ans, le plus récent étant celui de la Commission Pédagogie universitaire et documentation de l'ADBU $^{3}$, qui a publié en 2012 un Référentiel de compétences informationnelles, proposant une description fine de compétences, articulées à des pistes de formations possibles (ADBU, 2012). L'objectif visé par ce référentiel, qui s'adresse prioritairement aux étudiants de Licence, s'inscrit dans le droit fil de la «maîtrise de l'information » au service de la réussite des études.

\section{Une culture propre au monde de la recherche : l'information scientifique}

La culture de l'information scientifique ${ }^{4}$, proche des deux premières, s'en distingue pourtant sur deux plans: le public et le territoire, plus ciblés que ceux de la culture informationnelle. Son domaine comprend la connaissance, théorique et pratique, de tous les aspects de l'information scientifique, i.e. les modalités, circuits, acteurs et enjeux de l'écriture, de la production, de la publication, de l'évaluation scientifiques (par exemple les mécanismes du peer reviewing*, le Libre Accès, les blogs scientifiques, les outils de traitement et de visualisation des données, la problématique des Humanités Numériques, de la Science ouverte, l'évaluation de la recherche, etc.). La culture qui en est issue comprend un mixte de connaissances déclaratives (savoir ce qu'est une archive ouverte), de compétences techniques (utiliser un outil de cartographie de l'information), de savoir-faire informationnels (maîtriser les circuits de l'édition), de valeurs et de positionnements politiques (se positionner face au mouvement du Libre Accès), de représentations, de pratiques.

Si cette culture de l'information scientifique intéresse moins de monde que la culture informationnelle (qui concerne, littéralement, tous les usagers du Web), elle n'est plus réservée aux spécialistes et touche aujourd'hui de nombreux acteurs. Situé au carrefour du monde enseignant et de celui des bibliothécaires, qui y jouent un rôle essentiel insuffisamment reconnu, le monde de l'information scientifique est désormais un territoire commun, partagé par tous les acteurs de la recherche, du doctorant au chercheur expert, en passant par les professionnels de l'information, les ingénieurs, les décideurs. Autre mutation : cette culture, auparavant ancrée dans chaque discipline, est aujourd'hui plus transversale et la connaissance de son champ est devenue une nécessité pour les chercheurs, notamment les

\footnotetext{
${ }^{3}$ Association des Directeurs et personnels de direction des Bibliothèques Universitaires et de la documentation.

${ }^{4}$ Voir à ce sujet Schafer, 2014.
} 
doctorants de toutes disciplines (Malingre et Serres, 2011). Signalons enfin une évolution terminologique intéressante: autrefois appelée «Information scientifique et technique » (IST), elle se trouve aujourd'hui désignée sous le sigle ISN, pour Information scientifique numérique, marquant ainsi l'évolution vers la numérisation. Et l'un de ses enjeux de savoir est l'identification des compétences mobilisées, travail mené actuellement par le Segment 9 de la Bibliothèque scientifique numérique ${ }^{5}$ (BSN 9).

\section{La culture informatique, cruciale mais insuffisante}

Une autre culture essentielle est la culture informatique, qui englobe à la fois la connaissance de notions de base du «traitement automatique de l'information », et les multiples savoir-faire, recensés par exemple dans le $\mathrm{C}_{2} \mathrm{i}^{6}$. Au cœur de l'identité professionnelle des informaticiens, elle n'a cessé de s'étendre au-delà des sphères professionnelles, au fur et à mesure de l'extension du processus de numérisation à toutes les activités humaines, et le «grand public » est évidemment concerné, à différents degrés, par une culture informatique de base, par la compréhension minimale du fonctionnement d'un ordinateur, etc. Sur la spécificité de la culture informatique à l'université, deux enjeux nous semblent importants :

- l'acquisition des rudiments de cette culture devient un enjeu professionnel majeur pour la plupart des étudiants, appelés à s'insérer dans des univers professionnels complexes, où la maitrise, conceptuelle et pratique, des dispositifs* informatiques est cruciale. Or le constat est régulièrement fait, par différentes études ${ }^{7}$, que cette culture informatique reste notoirement insuffisante, limitée le plus souvent aux usages d'outils « grand public », plus ou moins bien maîtrisés ;

- l'appropriation d'une culture informatique, fondée sur la compréhension des principes et des logiques de l'informatique, représente également des enjeux de savoir et de société, sur lesquels les associations d'informatique et de nombreuses personnalités ont tiré la sonnette d'alarme depuis longtemps ${ }^{8}$. Vivre dans une société massivement informatisée, numérisée,

\footnotetext{
${ }^{5}<$ http://www.bibliothequescientifiquenumerique.fr/?BSN9-Formation-competences-et>

${ }^{6}$ Certificat informatique et internet. Voir sur : <http://c2i.education.fr/>

${ }^{7}$ Voir la synthèse de plusieurs études, par Laure Endrizzi, 2013.

${ }^{8}$ Sur les enjeux et la question d'un enseignement généralisé de l'informatique, voir notamment :

- le rapport de l'Académie des Sciences de mai 2013, disponible sur : <http://www.academiesciences.fr/activite/rapport/rads_0513.pdf>

- l'avis du Conseil National du Numérique sur l'enseignement de l'informatique en France, du 18 juin 2013 , disponible sur : <http://www.cnnumerique.fr/enseignementinformatique/>
} 
sans posséder le minimum de clés de compréhension de ces univers, aboutit inévitablement à des formes d'exclusion, d'incompréhension, voire d'asservissement à des logiques techniques devenues opaques. Si l'utilisation des réseaux sociaux ou d'un moteur de recherche ne nécessite pas de connaissances informatiques, la méconnaissance de leurs principes de fonctionnement participe d'un aveuglement face aux technologies, qui peut se payer au prix fort.

Élever le niveau des usages et des pratiques, pour permettre à la fois une meilleure insertion professionnelle et une compréhension critique des outils et des réseaux numériques : ce sont là deux enjeux majeurs d'une généralisation de la culture informatique à l'université. Enfin, un autre enjeu, plus théorique, est lié à cette culture : la réflexion sur les différences profondes séparant l'information, au sens de l'info-data, de l'information au sens de la connaissance, incarnée dans la culture informationnelle. Ces deux approches de l'information, inévitablement confondues dans les pratiques numériques, n'en demeurent pas moins irréductiblement différentes au plan épistémologique, autour de la question du sens. Alors que l'informatique, l'info-data, doit nécessairement évacuer la signification intrinsèque des données pour pouvoir les traiter de manière numérique, l'information «sociale », au sens de la connaissance, met la signification de ces données au premier plan. Et confondre la première avec la seconde aboutit au brouillage, largement répandu aujourd'hui, entre données et information, information et connaissance. Ces enjeux ne sont pas seulement théoriques, mais concernent aussi la formation des étudiants à l'exercice d'un esprit critique.

\section{L’insaisissable culture numérique}

La culture numérique est un vocable en vogue, dont le succès semble tout écraser sur son passage. À lire de nombreux rapports et articles, à croire les nombreux discours, souvent émerveillés, sur le numérique, la «culture » de celui-ci engloberait toutes les autres cultures, que nous nous astreignons à différencier ici. Or la notion même de culture numérique est problématique (voir notamment Simonnot, 2009). Si les trois précédentes cultures sont chacune portées par des histoires, des professions et des savoirs spécifiques, sur quels

- les nombreux rapports de l'association Enseignement Public et Informatique (EPI), disponibles sur : <http://www.academie-sciences.fr/activite/rapport/rads_0513.pdf> 
concepts, sur quels corpus de connaissances se fonderait cette culture numérique, nouvelle venue dans la galaxie des cultures de l'information?

Les diverses définitions et les référentiels qui fleurissent autour des compétences numériques illustrent la difficulté à définir et délimiter cette culture numérique, puisque les compétences numériques sont, alternativement, assimilées à :

- la seule culture informatique et à l'usage de l'ordinateur: par exemple pour Eurostat, l'office statistique de l'Union européenne, qui a publié en 2012 une enquête sur Les compétences numériques dans l'UE27 en chiffres ${ }^{9}$, celles-ci étant réduites à l'utilisation d'un ordinateur et de logiciels ;

- la seule culture et maîtrise du Web : par exemple pour la Fondation Mozilla, qui a publié récemment le «Web Literacy Standard», défini comme la «carte des compétences et aptitudes que Mozilla et notre communauté d'intervenants considèrent importantes pour s'améliorer à consulter, publier et participer sur le $\mathrm{Web}^{10} \gg$;

- l'usage de l'ordinateur, d'Internet, mais aussi de l'information, pour les référentiels du B2 $\mathrm{i}^{11}$ et du C2i ;

- plus largement un «usage sûr et critique des technologies de la société de l'information », pour le Parlement européen ${ }^{12}$;

- voire la culture informationnelle elle-même. Par exemple pour le gouvernement canadien : «il n'existe pas de définition convenue ni normalisée des compétences numériques. Toutefois, on s'entend pour dire qu'il s'agit de la capacité de repérer, d'organiser, de comprendre, d'évaluer, de créer et de diffuser de l'information par l'intermédiaire de la technologie numérique ${ }^{13} »$.

En France, plusieurs chercheurs ont souligné la difficulté à définir les compétences numériques, et s'interrogent sur la pertinence de la notion elle-même (Papi, 2012). En laissant de côté le débat théorique sur la notion elle-même, la culture numérique ne pourrait-elle se définir, de manière pragmatique, par les usages, les pratiques et les savoir-faire du numérique, incarnant à ce titre l'approche sociologique de la culture ? Comprenant à la fois les usages et les connaissances spontanées des outils, des réseaux et des productions liés au numérique, la culture numérique déborde ainsi la culture informatique, englobe une partie de la culture

\footnotetext{
${ }^{9}<$ http://europa.eu/rapid/press-release_STAT-12-47_fr.htm>

${ }^{10}<$ https://webmaker.org/fr/standard>

${ }^{11}$ Brevet informatique et internet : voir sur :<http://eduscol.education.fr/cid46073/b2i.html >

12 «Recommandation du Parlement et du Conseil européens du 18 décembre 2006 sur les compétences clés pour l'éducation et la formation tout au long de la vie ». Disponible sur : $<$ http://eur-lex.europa.eu/LexUriServ/LexUriServ.do?uri=OJ:L:2006:394:0010:0018:fr:PDF>

${ }^{13}<$ http://www.ic.gc.ca/eic/site/028.nsf/fra/00041.html>
} 
informationnelle et surtout intègre une dimension sociale forte, moins présente dans les autres.

Qu'elle soit considérée comme l'un des éléments de la galaxie des cultures de l'information, ou bien comme la nouvelle «mère de toutes les littératies ${ }^{14}$ », la culture numérique des étudiants et des jeunes chercheurs, assez massive aujourd'hui, pose sans doute un double enjeu à l'université, comme nous l'avons souligné plus haut :

- celui de la connaissance et surtout de la prise en compte, notamment pédagogique ${ }^{15}$, de cette culture numérique spontanée ;

- celui du développement d'une formation à la compréhension d'Internet, du Web et des outils numériques, de leur histoire et de leurs enjeux, d'une sensibilisation aux enjeux de «l'économie de l'attention », aux questions cruciales de l'identité numérique ${ }^{16}$, etc. Le champ des besoins et des thématiques est suffisamment riche pour construire, à partir de ces pratiques numériques spontanées, une véritable «culture numérique», scientifiquement fondée, à la fois théorique, pratique et surtout critique.

\section{Une culture des médias à redéfinir?}

La culture des médias est également difficile à définir, car elle repose sur une notion éminemment complexe. D’abord liée à la connaissance des médias de masse (presse, radio, télévision) et de l'image, cette culture a longtemps été l'apanage des Sciences de l'information et de la communication, des sociologues, des historiens des médias et bien sûr des journalistes. Dans l'enseignement secondaire, elle s'est incarnée dans les divers dispositifs de «l'éducation aux médias*» depuis plusieurs décennies. Mais sous l'effet de la numérisation et de la convergence de l'informatique, des télécommunications et de l'audiovisuel, le champ de la culture des médias n'a cessé de s'agrandir et de se redéfinir, selon un double mouvement :

- d'une part, une extension et un renouvellement en profondeur de ses objets d'étude : à la presse écrite et aux mass-medias traditionnels s'ajoutent désormais les réseaux sociaux, les forums, les blogs, etc. Si le Web en général peut être considéré comme un nouveau média,

\footnotetext{
${ }^{14}$ C'est notamment la perspective de BAWDEN, David D., « Digital Literacy », SciTopics, 2008. Disponible sur : $<$ http://www.scitopics.com/Digital_Literacy.html>

${ }^{15}$ Voir Laure Endrizzi. 2013.

${ }^{16}$ Voir notamment les travaux de Louise Merzeau (http://merzeau.net/tag/identite-numerique/) ou Julien Pierre (http://www.identites-numeriques.net/publications) sur l'identité numérique.
} 
alors le champ de la culture des médias connaît une extension à la mesure du Web, quasi illimitée ;

- d'autre part, la notion de média est elle-même extensive : on savait, depuis McLuhan dans les années 1960, que tout ensemble « sémio-socio-technique » pouvait être considéré comme un média, au sens littéral du terme ; tout objet, tout dispositif ayant une triple dimension sémiotique, sociale et technique, serait un média, comme le proclame fortement le récent rapport du Conseil supérieur de l'éducation aux médias de Belgique ${ }^{17}$.

Sans entrer ici dans les débats sur la définition de la culture des et de l'éducation aux médias, il nous semble évident que la connaissance et la pratique maîtrisée des médias (au sens classique du terme de dispositifs de production et de diffusion de l'information d'actualité) fait pleinement partie de la panoplie des compétences et des savoirs informationnels, fondamentaux pour les étudiants. Cette importance se constate notamment autour de ce point, crucial dans les études : la connaissance, toujours à approfondir, de la diversité des sources d'information, devenue infinie avec le Web, et des méthodologies de qualification et d'évaluation de ces sources ; savoir identifier, qualifier, analyser tel ou tel type de source, reconnaître le type de discours rhétorique tenu, évaluer sa crédibilité, sont des compétences relevant, selon nous, d'une culture des médias.

L'un des enjeux théoriques de cette culture des médias serait sa délimitation, ses relations, ses articulations d'avec les cultures, informatique d'une part, info-documentaire d'autre part. Les trois cultures se trouvent aujourd'hui tellement entremêlées dans les usages et les pratiques du web qu'il n'est pas facile de distinguer leurs compétences spécifiques. L'évaluation de l'information en ligne constitue ici un bon exemple de cette hybridation* des compétences, puisque le filtrage et la qualification des sources mobilisent diverses compétences, à la fois informatiques, informationnelles et médiatiques ${ }^{18}$.

\section{Une culture en émergence : la culture des données}

Ce tour d'horizon des cultures de l'information à l'université serait incomplet s'il ne mentionnait une culture en émergence, encore assez peu théorisée et dont le développement accompagne l'une des évolutions majeures actuelles du Web : la culture des données.

\footnotetext{
${ }^{17}<\mathrm{http}$ //www.educationauxmedias.eu/outils/brochures/csem/les_competences_en_education_aux_medias_cadre general >

${ }^{18}$ Sur cette question, voir Serres, 2012.
} 
«Web de données », Big Data, Open Data, ouverture des données de recherche, multiplication des outils de visualisation des données : la donnée est omniprésente sur le Web et, pour ce qui concerne notre propos, l'on voit apparaître de nouvelles compétences, générales et professionnelles, ainsi que de nouveaux métiers (les «data scientists », les «data managers »).

Certes, le traitement et la production de données n'ont pas attendu le Web et les données sont depuis longtemps au cœur du travail des chercheurs. Et la question des compétences nécessaires au traitement de ces données (depuis l'utilisation maîtrisée d'un tableur jusqu'à celle d'un outil de «fouille de données ») n'a rien de nouveau. Mais l'accès à des masses croissantes de données de toutes sortes, produites par le phénomène du «Big Data », ces quantités phénoménales de données circulant sur Internet, concerne déjà de très nombreux chercheurs et doctorants, qui les utilisent pour leurs travaux de recherche.

La nouveauté réside, comme toujours avec Internet, dans un triple changement d'échelle :

- dans la quantité de données désormais accessibles librement ;

- dans la diversification, la multiplication, la puissance des outils de traitement ;

- dans l'acuité et la multiplicité des enjeux de toutes sortes: sociétaux (utilisation commerciale des données personnelles), politiques (surveillance généralisée), scientifiques (questions posées par l'ouverture des données de recherche), etc.

Apparaissent aussi de nouveaux enjeux de savoirs : quelles sont les nouvelles compétences nécessaires aujourd'hui pour la collecte, le traitement, l'analyse, la visualisation, l'interprétation de ces masses de données ? Un récent billet de blog, sur le site de la FING (Fondation Internet nouvelle génération), identifiait ainsi «7 champs de compétences mobilisées » (Brugière, 2013) dont la liste est intéressante à citer : Lecture-écriture, Culture numérique, Information-documentation-communication, Droit, Statistique, Informatique, Design, Graphisme. Sans surprise, nous y trouvons trois cultures de l'information, et d'autres plus spécifiques (droit, statistique, design). Nul doute que ce mixte de compétences est appelé à se développer fortement à l'université, dans tous les cursus et à tous les niveaux.

\section{Trois défis à relever}

Le défi de la multiplicité : multiplicité des cultures, des relations entre ces cultures, avec des délimitations parfois délicates, multiplicité des compétences mobilisées, des acteurs, des problématiques, des objets de recherche, des enjeux... Cette multi-dimensionnalité des cultures de l'information à l'université nécessiterait un important chantier de recherche et de 
réflexion théorique, pour penser ce mixte de cultures et le traduire ensuite dans des dispositifs de formation. Au lieu de placer toutes les cultures et compétences nécessaires sous la seule égide d'une culture numérique fourre-tout, il importerait de remettre de la clarté dans la confusion des cultures de l'information, générée par la révolution numérique : savoir utiliser un média ne relève pas de l'informatique, la méthodologie documentaire n'est pas morte avec les réseaux sociaux, etc. Si le même support unifie toutes les pratiques, il ne saurait faire disparaître les cultures spécifiques. Ce défi de la multiplicité impliquerait ainsi une mise à plat des compétences et des cultures, mobilisées dans les interactions numériques.

Le défi de leur articulation avec les disciplines académiques : quelle autonomie de chacune de ces cultures de l'information par rapport aux disciplines traditionnelles ? Si la question n'est pas nouvelle pour la culture info-documentaire, elle mérite sans doute d'être actualisée pour les autres cultures de l'information, toutes transversales par nature. La question posée étant celle de la possibilité (épistémologique et académique) d'une «culture informationnelle commune » à toutes les disciplines.

Enfin, le défi des outils, qui jouent un rôle clé, aussi bien dans ces cultures de l'information que dans toutes les disciplines. Face à l'extraordinaire multiplicité des outils (de recherche, de veille, de traitement, de visualisation, de publication, de mémorisation, d'annotation, d'écriture, de lecture, de travail collaboratif, de traitement des données, etc.), la compréhension et la maîtrise de ces outils numériques sont devenues à la fois une condition clé de la recherche, et une condition de succès dans les études.

Références bibliographiques

ADBU, «Référentiel de compétences informationnelles. Pour réussir son parcours de formation dans les établissements d'enseignement supérieur », Paris, ADBU, décembre 2012. http://fr.calameo.com/read/0020941243908e7791f54

BALTZ, Claude, «Une culture pour la société de l'information? Position théorique, définition, enjeux », Documentaliste - Sciences de l'information, vol. 35, n 2, 1998, p. 75-82. 
BrugiÈRE, Amandine, «Y a-t-il des compétences "Data" spécifiques? », Finglive, [en ligne], 2 septembre 2013. Disponible sur <http://fing.tumblr.com/post/60060091542/y-a-t-il-descompetences-data-specifiques>

CONSEIL SUPÉRIEUR DE L'ÉDUCATION AUX MÉDIAS et BOUMAL, Michel (dir.), Les compétences en éducation aux médias : un enjeu éducatif majeur. Cadre général, rapport de synthèse, Bruxelles, Conseil supérieur de l'éducation aux médias, septembre 2013. Disponible sur: $<$ http://www.educationauxmedias.eu/sites/default/files/files/CompetencesEducationMedias_ Web.pdf>

Coulon, Alain, «Un instrument d'affiliation intellectuelle. L'enseignement de la méthodologie universitaire dans les premiers cycles universitaires », Bulletin des Bibliothèques de France, $\mathrm{n}^{\circ}$ 1, 1999, p. 36-42. Disponible sur : <http://bbf.enssib.fr/consulter/bbf-1999-01-0036-005>

ENDRIZZI, Laure, «Enseignants et étudiants: des visions et des pratiques décalées », Pédagogie numérique pour l'économie et la gestion, [en ligne] séminaire international, Paris La Défense, 23-24 mai 2013, Institut Français de l'Éducation, 2013. Disponible sur : <http://laure-endrizzi.net/wp-content/uploads/2013/06/Diapo-endrizzi-Aunege-mai-2013.pdf>

MALINGRE, Marie-Laure et SERRES, Alexandre, « Une culture informationnelle commune aux doctorants ? Le pari de Form@doct», in DENECKER, Claire, DuRAnd-BARTHEZ et Manuel (dir.), La formation des doctorants à l'information scientifique et technique, Villeurbanne, Presses de l'ENSSIB, 2011. p. 53-67.

PAPI, Cathia, «Des référentiels à la validation des compétences numériques : questionnements et dispositifs », Questions Vives [en ligne], volume7, n 17, 2012. Disponible sur : http://questionsvives.revues.org/986

SCHAFER, Valérie (dir.), Information et communication scientifiques à l'heure du numérique, Paris, CNRS Éditions, 2014, coll. « Les Essentiels d'Hermès ». 
SERRES, Alexandre, «Un exemple de translittératie : l'évaluation de l'information », Les edossiers de l'audiovisuel, INA, janvier 2012, L'éducation aux cultures de l'information. Disponible sur : <http://www.ina-expert.com/e-dossier-de-1-audiovisuel-1-education-auxcultures-de-1-information/un-exemple-de-translitteratie-l-evaluation-de-l-information-surinternet.html>

SimONNOT, Brigitte, « Culture informationnelle, culture numérique : au-delà de l'utilitaire », Cahiers du numérique, vol. 5, $\mathrm{n}^{\circ}$ 3, septembre 2009, p.25-37.

Thomas, Sue et al., «Transliteracy: Crossing divides », First Monday [en ligne], volume 12, $\mathrm{n}^{\circ}$ 12, 3 Décembre 2007. Disponible sur :

< http://www.uic.edu/htbin/cgiwrap/bin/ojs/index.php/fm/article/view/2060/1908> 